\title{
Reproducing the Proximal Femur Anatomy: 3D Preoperative Planning and Custom Cutting Guides
}

\author{
Tyler A. Luthringer and Jonathan M. Vigdorchik
}

\section{Key Points}

- Custom femoral cutting guides may increase the accuracy and precision of the femoral neck osteotomy based on patient-specific targets from 3D preoperative planning.

- The level and angle of the neck cut affects final stem height and coronal alignment, while proximal femur anatomy and canal morphology influence femoral stem version in uncemented designs.

- Available femoral PSI systems only control the level and angle of the osteotomy and do not yet guide stem version, although they provide a useful preoperative reference to help decision making.

- Additional research is necessary to confirm the efficacy of femoral guidance PSI in achieving targeted stem height, position, and version, as well as reveal the effect on clinical outcomes compared to traditional techniques.

\section{T. A. Luthringer}

NYU Langone Orthopedic Hospital,

NYU Langone Health, New York, NY, USA

e-mail: Tyler.Luthringer@nyulangone.org

J. M. Vigdorchik $(\bowtie)$

Hospital for Special Surgery, New York, NY, USA

e-mail:VigdorchikJ@HSS.edu

\subsection{What Is the Rationale?}

Successful outcomes of total hip arthroplasty (THA) depend upon patient-specific factors, surgical technique, and appropriate implant selection. Proper surgical technique requires meticulous preoperative templating, followed by accurate and precise component positioning, a modifiable risk factor which may prevent poor clinical function following THA. Restoration of native hip biomechanics serves to optimize implant wear and THA stability. Closely approximating native hip biomechanics also avoids abductor insufficiency, limb-length inequality, and early construct failure. A key challenge to accurate component placement includes accommodating for variations in individual patient anatomy, functional spinopelvic mobility, and intraoperative positioning. Threedimensional (3D) preoperative templating and patient-specific instrumentation (PSI) have emerged to enhance the surgical precision of bone resection and individualize component placement in THA.

Ideal femoral component position restores leg length, femoral offset, and femoral version. Conventional templating on two-dimensional (2D) anteroposterior (AP) pelvic radiographs is often limited by inaccurate magnification and variable rotational alignment of the proximal femur. Femoral offset may be underestimated on AP pelvis radiographs due to the projectional 
effects of femoral anteversion and external rotation contractures that may be present in latestage osteoarthritis [1]. Uncemented stem designs follow the medullary canal from the aperture of the neck cut to achieve mediolateral metaphyseal and distal diaphyseal fixation [2]. The shape of the proximal femur thereby influences the final stem anteversion and coronal alignment [3]. Additionally, femoral canal morphology varies significantly at different planes of axial resection due to the complex anatomy of the proximal metaphyseal bone [4]. As a result, the angle and level of the osteotomy respectively influence the anteversion and varus/valgus alignment of the femoral component [4]. Freehand femoral osteotomy is accurate to within $4 \mathrm{~mm}$ of conventionally templated targets in only $87 \%$ of cases, which may introduce significant variability in final stem height and position and ultimately result in alterations in limb length [4].

Three-dimensional templating optimizes stem size and position to achieve optimal metaphyseal loading for fixation and anatomical restoration. The addition of axial imaging mitigates the shortcomings of 2D coronal templating while the use of custom femoral cutting guides helps to limit variability in surgical technique and minimize outliers of leg length, offset, and version. Femoral guidance PSI systems additionally incorporate kinematic simulation of the hip, pelvis, and lumbar spine in preoperative planning to assess impingement-free range of motion throughout functional extremes of posture [5]. Currently, there are four commercially available PSI hip systems, two of which include femoral guides (MyHip from Medacta and OPS $^{\text {TM }}$ from Corin). As of early 2019, the OPS ${ }^{\text {TM }}$ is the only femoral PSI system approved by the Food and Drug Administration (FDA) for use in the United States. While the OPS ${ }^{\mathrm{TM}}$ will serve as the primary example system for the purposes of this chapter, the methodology and implementation of each system is generally the same. As the roles of 3D preoperative planning and the intraoperative use of femoral PSI guides are intimately intertwined, the indication for and potential benefit of their utilization is mutually considered.

\subsection{What Are the Best Indications?}

Relatively healthy bone stock is required for rigid fixation of PSI guides intraoperatively [5]. Femoral guides are fixed into place via two interosseous pins prior to performing the osteotomy. If the integrity of pin fixation is compromised by poor bone quality, or if the guide cannot be reliably secured in the intended position, the accuracy of the neck cut will be affected. The adjunct of 3D preoperative templating and femoral guidance PSI may be particularly valuable in young, active patients, in patients prone to postoperative instability, in patients suspected to have excessive native femoral ante- or retroversion or extreme neck-shaft angles, and for surgeons who employ minimally invasive approaches to the hip.

Young, active patients: In young, active THA patients, restoration of native hip biomechanics is not only important to increase construct longevity but also for the maintenance of physiological hip soft-tissue balance and native hip joint kinematics. Patients with high levels of activity are more likely to notice small inconsistencies between their native and artificial hips as well as overall limb lengths, placing them at greater risk for postoperative dissatisfaction. Femoral guidance PSI can help to limit postoperative limblength outliers in younger patients who may be sensitive to such minimal discrepancies. Younger, active patients also more regularly assume positions of extreme hip flexion and extension compared to their older counterparts. Femoral PSI systems combine 3D anatomic model reconstruction with dynamic spinopelvic imaging to plan for optimal component position in a variety of functional positions. This kinematic simulation estimates the magnitude and direction of hip joint reaction forces across extreme ranges of motion. Imparting this knowledge to surgeons preoperatively allows for consideration of potential wear rates in young patients and impingement risk in more active candidates (such as those who wish to return to yoga or extreme sports).

Instability-prone patients: A growing body of evidence has questioned the universal application of traditional acetabular "safe zones" in all 
patients undergoing THA. Certain populations have been identified to be at increased risk for dislocation secondary to "functionally" malpositioned acetabular components. Arthroplasty surgeons have begun to mitigate this risk by tailoring traditional cup position to individual spinopelvic kinematics. While evidence supports that candidates with limited spinopelvic mobility may particularly benefit from patient-specific cup placement, the implications of femoral component position on THA stability are relatively less well understood. Nonetheless, due to the known effect of the combined anteversion of the cup and stem in conferring impingement-free range of motion [3], complementary use of acetabular and femoral PSI may be considered in patients with stiff spines or prior lumbar fusions who are at higher risk for dislocation. Similar to that in young, active patients, the preoperative aspect of dynamic spinopelvic imaging and kinematic simulation is of equal importance to the intraoperative implementation of planned component positioning with PSI in this population.

Femoral neck variants: Variation in femoral canal morphology in the periaxial plane of resection and overall proximal femur anatomy influences the final version and coronal alignment of the femoral stem with uncemented implant designs [3, 4]. Aberrant femoral neck version or neck-shaft angle alters the habitual anatomic orientation of the osteotomy relative to the surgeon and patient. This may introduce significant variability in the angle and level of a freehand neck cut relative to templated targets. Provided the extent of the anatomy does not preclude adequate guide fixation, there is a theoretical advantage to femoral guidance PSI in patients with significant femoral neck ante- or retroversion, as well as coxa valga or vara. Custom femoral cutting guides may help to reproduce the height and plane of the intended osteotomy and more reliably achieve targeted femoral stem level and position in these challenging cases.

Minimally invasive approaches: Patientspecific instrumentation may be a particularly valuable tool in improving the precision of femoral neck osteotomies for surgeons who employ minimally invasive approaches in THA. In mini- mally invasive hip surgery, limited exposure of the operative field leaves bony landmarks less accessible. As the femoral neck osteotomy is typically referenced from the lesser trochanter, use of femoral guidance PSI may reduce the margin of error and obviate the need for intraoperative imaging when performing the femoral neck cut with limited exposure.

\subsection{What Is the Process?}

3D preoperative planning: PSI systems require preoperative imaging with computed tomography (CT) or magnetic resonance imaging (MRI) to create the patient-specific joint model as well as make a template of the custom cutting guides and implants. A 3D computer model is generated and utilized to virtually plan the position and size of the prosthesis. In addition, functional spinopelvic imaging in sitting/standing positions may be used in some systems to define the limits of hip extension and flexion and assess the effect of chosen component position on implant and bony impingement in those functional positions. Inputs from these functional radiographs are also used for a kinematic simulation that shows the direction and magnitude of hip joint reactive forces throughout range of motion [6]. Preoperatively, surgeons may view the expected alterations in bearing contact mechanics at different component positions. Final planned implant positions may ultimately be tailored to the surgeon's preference for each patient prior to instrumentation manufacturing.

Manufacturing: Custom cutting guides are designed to fit and complement the native anatomy using bony or cartilage landmarks on CT or MRI, respectively. The guides are produced by either selective laser sintering or 3D printing and sterilized for delivery to the surgeon's center. Both posterior and anterior femoral cutting guides are offered to the surgeon to best suit the preferred surgical approach (dislocating versus in situ neck cut, respectively) (Fig. 9.1). The whole process from preoperative imaging to guide acquisition generally takes between 3 and 8 weeks [5]. 
Fig. 9.1 Custom cutting guides for femoral neck osteotomy

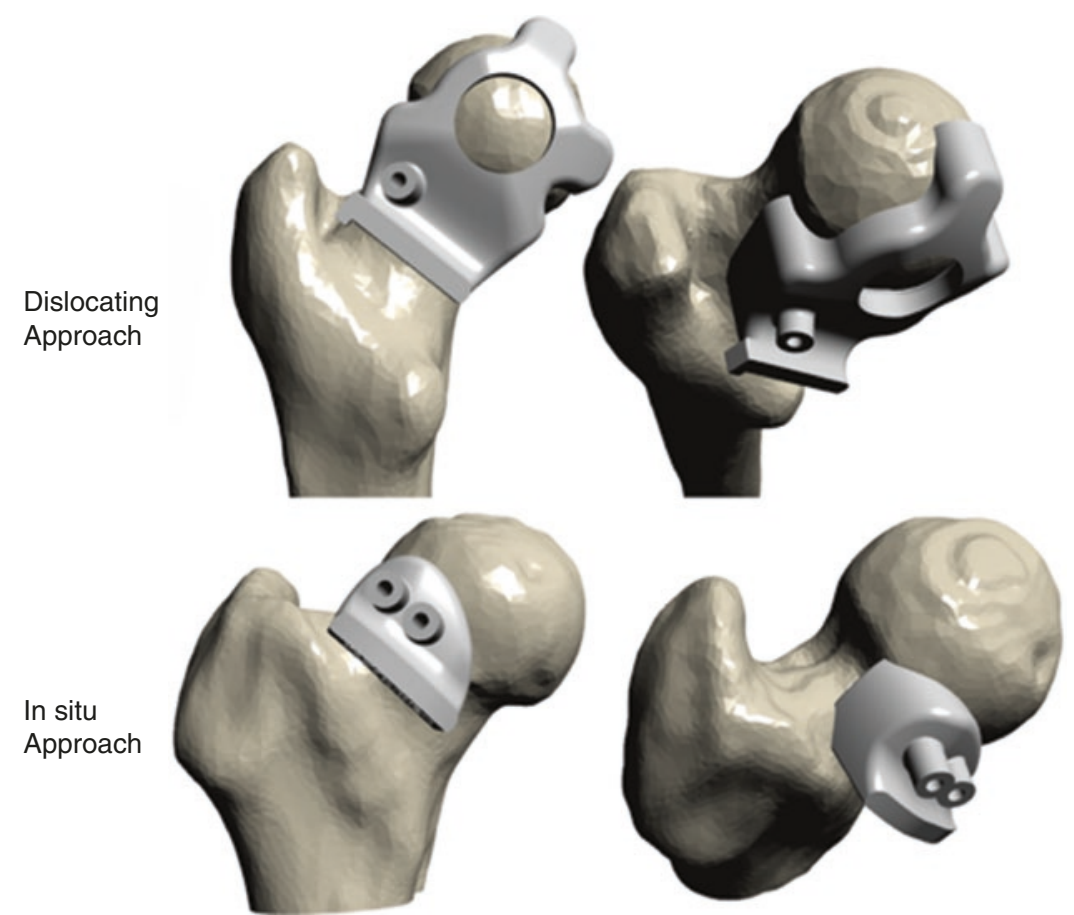

Intraoperative implementation: Following routine exposure intraoperatively, the femoral guide is positioned on the head-neck junction and secured in place with a pin. The osteotomy is done with a standard oscillating saw along an open capture feature of the guide, which controls the level and angle of the neck cut but does not directly guide stem version.

\subsection{What Are the Specific Related Complications?}

There have been no reports of specific complications related to the use of femoral PSI in THA in the literature; however, most published series have been limited to approximately 30 patients or less $[5,7,8]$. No significant differences in blood loss or operative time have been shown to exist between patient-specific and conventional instrumentation. Comparisons to date have been limited to acetabular PSI and are undoubtedly influenced by surgeon experience [5]. Theoretical complications of currently available femoral guidance PSI include improper guide-anatomy fit, inadequate or loss of guide fixation, and iatrogenic fracture caused by pin fixation or guide misuse.

Use of PSI and 3D custom cutting guides requires the patient to undergo a $\mathrm{CT}$ scan in the preoperative period, and thus, it is associated with additional time, cost, and radiation exposure (unless using an MRI-based PSI system). The effective dose of radiation associated with the CT scan has been determined to be $2.8 \mathrm{mSv}$, which is similar to average annual background radiation exposure $[9,10]$. There is currently no evidence to support the superiority of either CT or MRI for the creation of PSI in THA. The preferred PSI system determines which imaging modality is employed.

\subsection{What Is the Supporting Clinical Evidence?}

Due to the recent advent of femoral guidance PSI in THA, there are limited published reports on its clinical use yet available in the literature. Generic osteotomy guides have been shown to improve 
the accuracy of femoral neck resection height and limit postoperative limb length discrepancy (LLD). Yang et al. developed a set of six osteotomy guides with 1-mm interval height differences suitable for use on a variety of femoral neck configurations [11]. In 48 patients randomized to undergo THA with and without use of the guides, the mean average differences in femoral neck resection height were $0.84 \mathrm{~mm}$ and $1.69 \mathrm{~mm}$, respectively [11]. The mean postoperative LLD was $5.45 \mathrm{~mm}$ among the guide group compared to $13.37 \mathrm{~mm}$ in the control group [11]. Ito et al. conducted an initial feasibility study on the use of PSI for femoral stem placement in THA. Using CT scan data for 3D planning and computer modeling software, the authors designed and manufactured 10 patient-specific femoral osteotomy guides for individual clinical use [7]. Compared to their preoperative targets, postoperative CT demonstrated the mean accuracy of femoral stem tilt, varus/valgus, and anteversion to be $2.1^{\circ} \pm 4.1^{\circ}, 1.0^{\circ} \pm 0.7^{\circ}$, and $4.7^{\circ} \pm 1.2^{\circ}$ with use of their PSI guides, respectively [7].

Early clinical data have supported the efficacy of the commercially available OPS proximal femoral cutting guide in achieving accurate osteotomies $[8,12,13]$. In an analysis of 33 patients, early use of the OPS femoral cutting guide by two surgeons was found to be clinically accurate within $1 \mathrm{~mm}$ of the planned osteotomy level in $85 \%$ of cases, with a mean difference of $0.7 \mathrm{~mm}$ between achieved osteotomy levels and templated targets [12]. In a subsequent series, 100 patients underwent posterior THA by one of three surgeons; use of PSI yielded femoral neck osteotomies within $1 \mathrm{~mm}$ and $2 \mathrm{~mm}$ of the preoperative plan in $83 \%$ and $96 \%$ of cases, respectively [13]. The mean difference between the planned and achieved osteotomy level was $0.3 \mathrm{~mm}$, with a maximum reported error of $4 \mathrm{~mm}$ [13]. Schneider et al. subsequently analyzed the radiographic outcomes of 30 patients who underwent uncemented PSI-THA via the minimally invasive direct superior approach [8]. A total of 29 of 30 osteotomies were found to be within
$3 \mathrm{~mm}$ of planned height [8]. In each of these studies, the achieved level of the femoral neck osteotomy at the medial calcar was compared to the planned level of resection using a 3D/2D matching analysis (Mimics X-ray module, Materialise, Belgium), and all patients received a Trinity/ TriFit TS uncemented THA (Corin, Cirencester, UK) $[8,12,13]$.

A single-center pilot study of 100 patients analyzed restoration of femoral head center of rotation using the OPS 3D femoral planning. In this series, the mean differences in planned and achieved head height, medial offset, and anterior offset were $0.9 \mathrm{~mm},-0.9 \mathrm{~mm}$, and $3.2 \mathrm{~mm}$, respectively [14]. The resulting $3 \mathrm{D}$ change in planned and achieved head center was $4.4 \mathrm{~mm}$; changes in anterior offset were strongly correlated to differences in achieved stem anteversion compared to planned targets $\left(16.3^{\circ} \mathrm{vs}\right.$. $10.5^{\circ}$, respectively) [14]. While there was no comparison group, the authors conclude that use of 3D templating and PSI femoral guides accurately reproduce femoral center of rotation.

The OPS 3D planning software has also been evaluated for the sizing accuracy of the Trinity/ TriFit TS components. In a consecutive series of 49 THAs, $92 \%$ of implanted TriFit TS femoral stems were within one size of that predicted, and use of standard or high-offset stems was predicted correctly in $80 \%$ of cases [15]. Variability in final stem offset chosen was largely attributed to the extent of medialization of the acetabular component.

Despite reports of the operative reproducibility of PSI femoral guidance, there have been no published studies on the clinical or functional outcomes following use of the technology in THA. Commercially available instrumentation has been validated in its ability to aid surgeon execution of femoral neck osteotomies at the desired (templated) level based on radiographic outcomes. However, data that directly compares the accuracy of femoral guidance PSI to conventional techniques have not yet been reported. Further study needs to address whether these 
radiographic outcomes correlate with functional and clinical outcomes in patients undergoing THA with femoral PSI. The body of literature on femoral guidance PSI in THA will undoubtedly grow with the continued use of this technology and appropriate patient follow-up.

\subsection{Convincing Arguments: Why Recommend?}

Variable magnification and out-of-plane rotations limit the reliability of 2D X-rays for accurate templating. Native proximal femur anatomy tends to guide femoral component alignment with use of uncemented stem designs [2, 3], while the level and angle of the femoral neck cut has been shown to influence final stem height and position [4]. Undersized femoral components can lead to a limb shortening, stem subsidence, and instability secondary to insufficient offset. Oversizing the femoral component may restrict hip motion, cause excessive limb lengthening, and increase the risk of intraoperative fracture. Three-dimensional preoperative planning includes assessment of femoral version and proximal canal morphology to more reliably measure true native offset and predict implant size. Patient-specific instrumentation may enhance the precision and accuracy of the osteotomy technique to increase consistency between final stem position and templated targets. Together, use of patient-specific 3D preoperative templating and custom femoral guides can help minimize outliers of limb length, offset, and stem version, and ultimately benefit clinical outcomes.

Limb length discrepancy (LLD) is the most common reason for patient dissatisfaction and litigation following THA [16, 17]. Errors in limb length are due to improper femoral stem positioning in $98 \%$ of cases [18]. Although conventional templating and techniques may effectively keep LLD to $<10 \mathrm{~mm}$ in $97 \%$ of cases [19], evidence has shown that discrepancies $>5 \mathrm{~mm}$ are likely to be perceived by patients [20,21]. Greater discrepancies may result in the need for a shoe lift, potentiate back and radicular pain, increase pelvic obliquity, and cause implant failures such as instability, accelerated wear, and early loosening [16]. Residual LLD after THA has been associated with abnormal hip biomechanics, alterations in gait, and worse functional outcome scores $[18,20,22,23]$. The extent of these adverse clinical effects is relative to the magnitude of the LLD, tends to be patient-specific, and may or may not improve with time elapsed from surgery. Younger, more active patients are far less likely to tolerate any significant alterations in limb length following THA; they may particularly benefit from PSI.

Femoral offset is the horizontal distance from the center of rotation of the femoral head to a line bisecting the anatomic axis of the femur. This length is underestimated by up to $20 \%$ on 2D radiographs [24]. In THA, femoral offset is influenced by the coronal (varus/valgus) alignment and anteversion of the stem, as well as the neck-shaft angle of the implant design. Global offset, defined as the sum of acetabular offset and femoral offset, should invariably be restored in THA [25]. Reductions in global offset result from imbalanced positioning of both the acetabular cup and femoral stem [18, 26]. While medialization of the acetabular cup (a decrease in acetabular offset) serves to reduce joint reactive forces and optimize bearing surface wear, a compensatory increase in femoral offset is required to maintain soft-tissue tension and avoid impingement. Decreasing offset in THA may result in abductor weakness, altered gait, and instability [16, 24, 27]. A significant increase in offset may contribute to lateral-sided hip pain and greater trochanteric bursitis. Accordingly, failure to restore offset has been shown to decrease patient satisfaction, quality of life, and yield worse functional outcomes $[16,25]$.

Acetabular and femoral component positions are mutually important for THA stability. Dorr et al. have suggested that the combined anteversion of the cup and stem (optimal range $25-50^{\circ}$ ) is more important in conferring impingement- 
free motion and constructing stability than acetabular "safe zone" position alone [3]. As our appreciation of the importance of combined anteversion continues to grow, the demand for accurate femoral component delivery systems such as PSI is likely to rise. Presently, femoral PSI guides control only the level and angle of the neck cut and do not yet control stem version. Nonetheless, accurate cup positioning and assessment of femoral version and canal morphology with available PSI-THA systems is beneficial for the surgeon in achieving combined anteversion targets.

Patient-specific instrumentation is a novel modality designed to enhance surgical technique and improve the accuracy of component positioning in THA. Currently available systems offer an alternative approach to patient-specific THA for surgeons without access to computer navigation or robotic-assisted platforms. Ongoing research will determine the efficacy of custom femoral cutting guides in reproducing desired femoral component position, as well as the effect on patient-reported and functional outcomes.

\subsection{Case Example}

The following section offers a generic visual guide to further explain the 3D preoperative templating for femoral component position using the OPS $^{\mathrm{TM}}$, Corin Group. An example of a final OPS Plan for PSI-THA is shown in conclusion.

\subsubsection{Length Planning}

- The planned position of the entire femoral component (stem/head) is measured relative to the tip of the greater trochanter to reproduce the native femoral head center (Fig. 9.2). In patients who have undergone a contralateral THA, the stem/head combination is planned to match the head center of the contralateral prosthesis (Fig. 9.2).
- Postoperative length change is measured in the superior-inferior direction from the templated center of rotation of the liner (green) to the templated prosthetic head center (pink), and is compared to the preoperative state. The stem height is planned such that the osteotomy level is at least $5 \mathrm{~mm}$ superior to the lesser trochanter (unless otherwise specified) (Fig. 9.3).

\subsubsection{Offset Planning}

- As the acetabular component is often medialized, planned femoral offset of the stem/head combination is generally increased to maintain global offset.

- Center of rotation medialization is measured from the native femoral head center to the templated center of rotation of the liner. Femoral offset is measured from the native femoral head center to the templated prosthetic head center (Fig. 9.4). Offset is the planned overall change in hip offset when the femoral prosthesis is concentrically reduced into the acetabular component $($ Offset $=$ femoral offset - Center of rotation medialization) (Fig. 9.4).

\subsubsection{Stem Version Planning}

- The femoral stem position is templated to reproduce the native femoral head center in the axial or transverse plane.

- Native femoral version is measured as the angle subtended by axis of the native femoral neck and the line tangent to the posterior condyles of the knee viewed down the long axis of the femur (Fig. 9.5).

Stem version is the angle between the axis of the neck of the femoral stem and the line tangent to the posterior condyles of the knee, again viewed down the long axis of the femur (Fig. 9.6). 


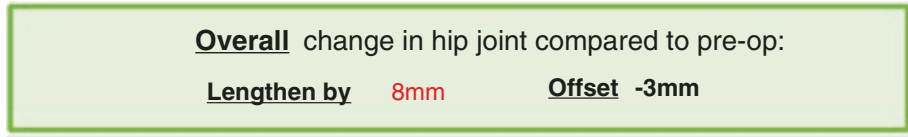

Femoral offset increased by $0 \mathrm{~mm}$

Centre of rotation medialised by $3 \mathrm{~mm}$

Warnings (see page 3 for reference)

- Lengthening by $8 \mathrm{~mm}$ compared to pre-op [1]

- Step cut is required [5]

- Planned femoral head centre is $7 \mathrm{~mm}$ higher than native head centre [6]

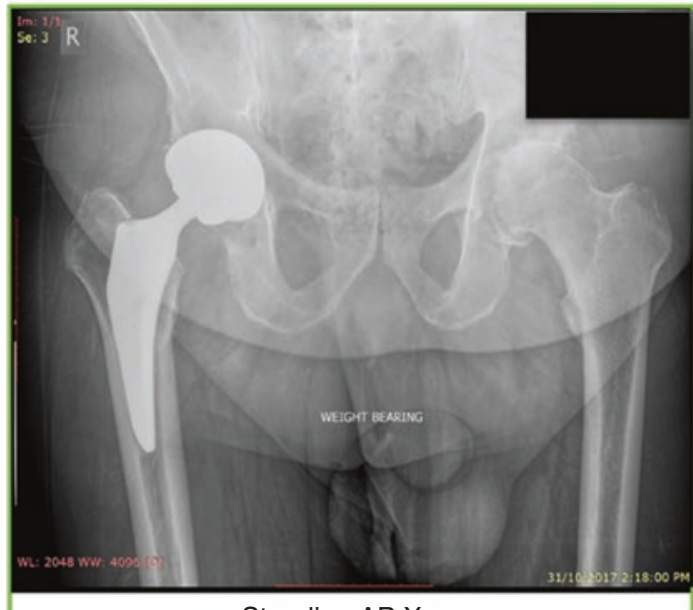

Standing AP X-ray

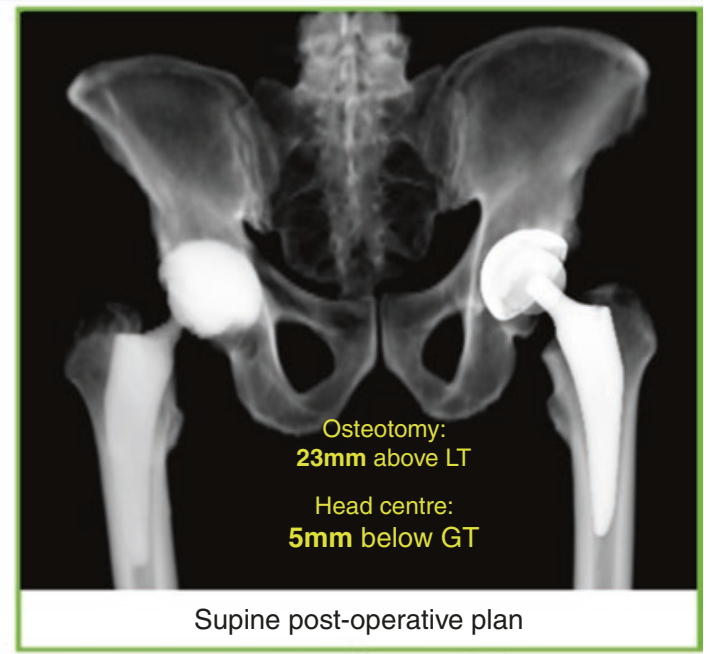

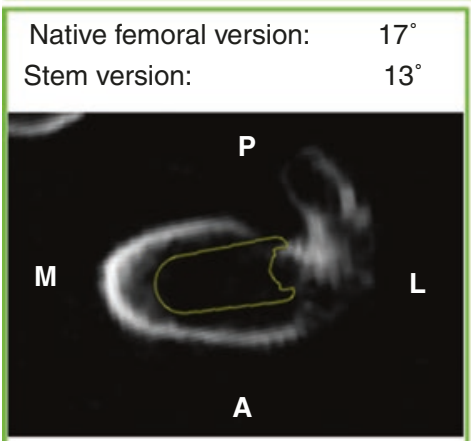

Section in osteotomy plane
Cup orientation when supine: $44^{\circ} / 22^{\circ}$

Cup: 56 Trinity

Stem: \#9 Std TriFit

Head: $\mathbf{3 6}+\mathbf{4}$

The default stem placement is planned to reproduce the native femoral head centre in the transverse plane, unless specified otherwise. See page 2 for details.

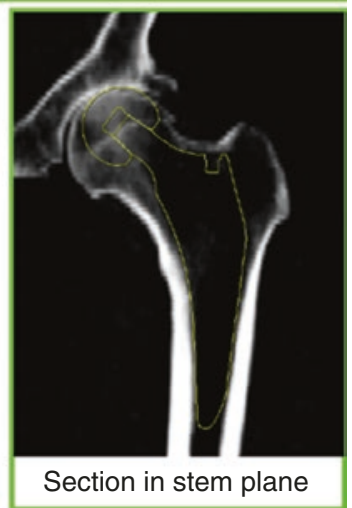

Fig. 9.2 Example of OPS plan targeted to contralateral implant head height 
Fig. 9.3 Predicted length change and osteotomy relative to the lesser trochanter
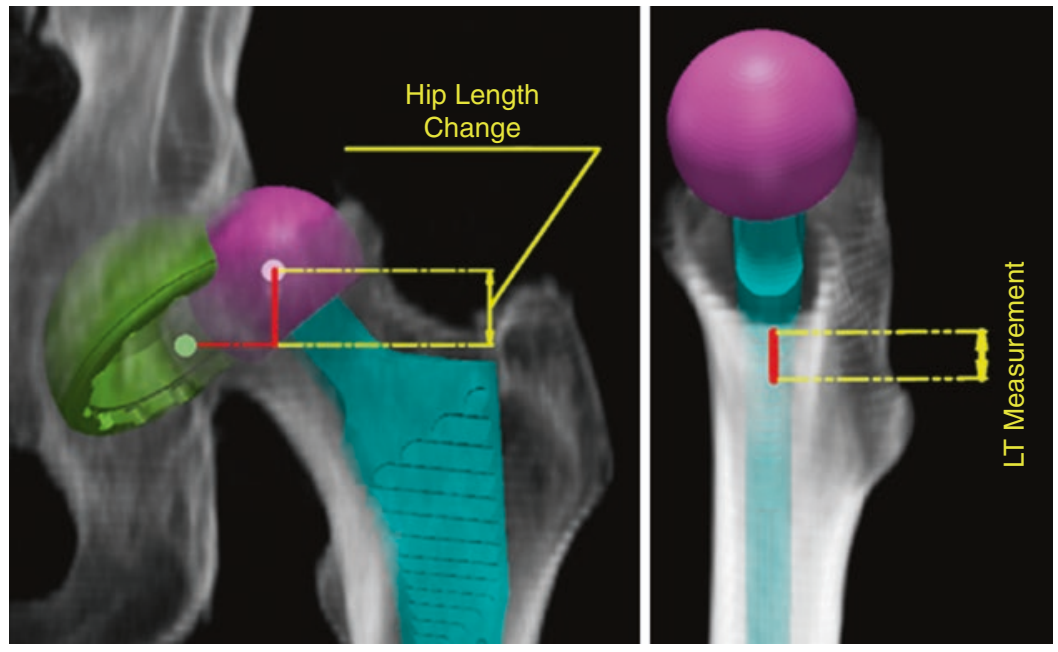

Fig. 9.4 Predicted change in global offset
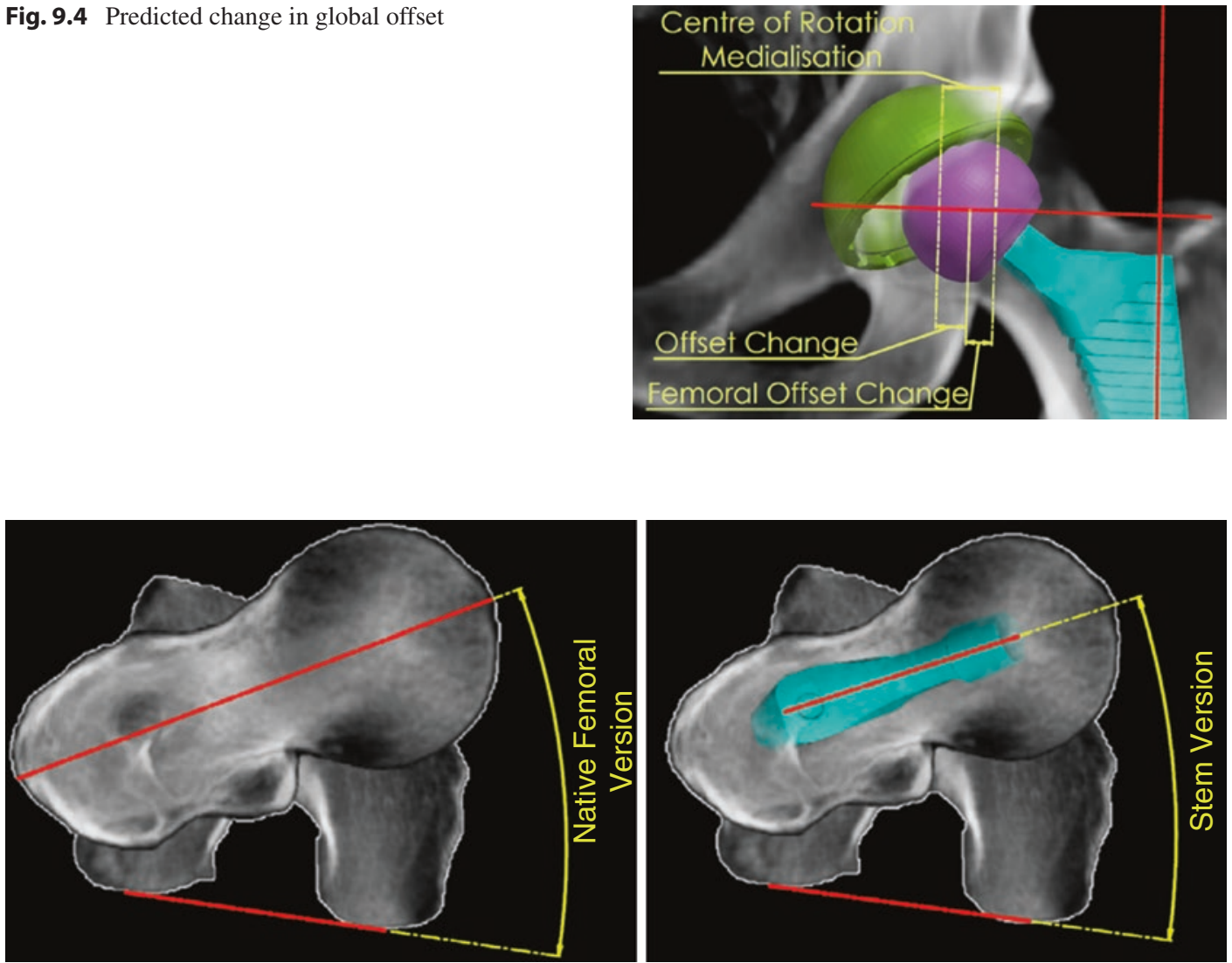

Fig. 9.5 Planned femoral stem version based on native femoral version 

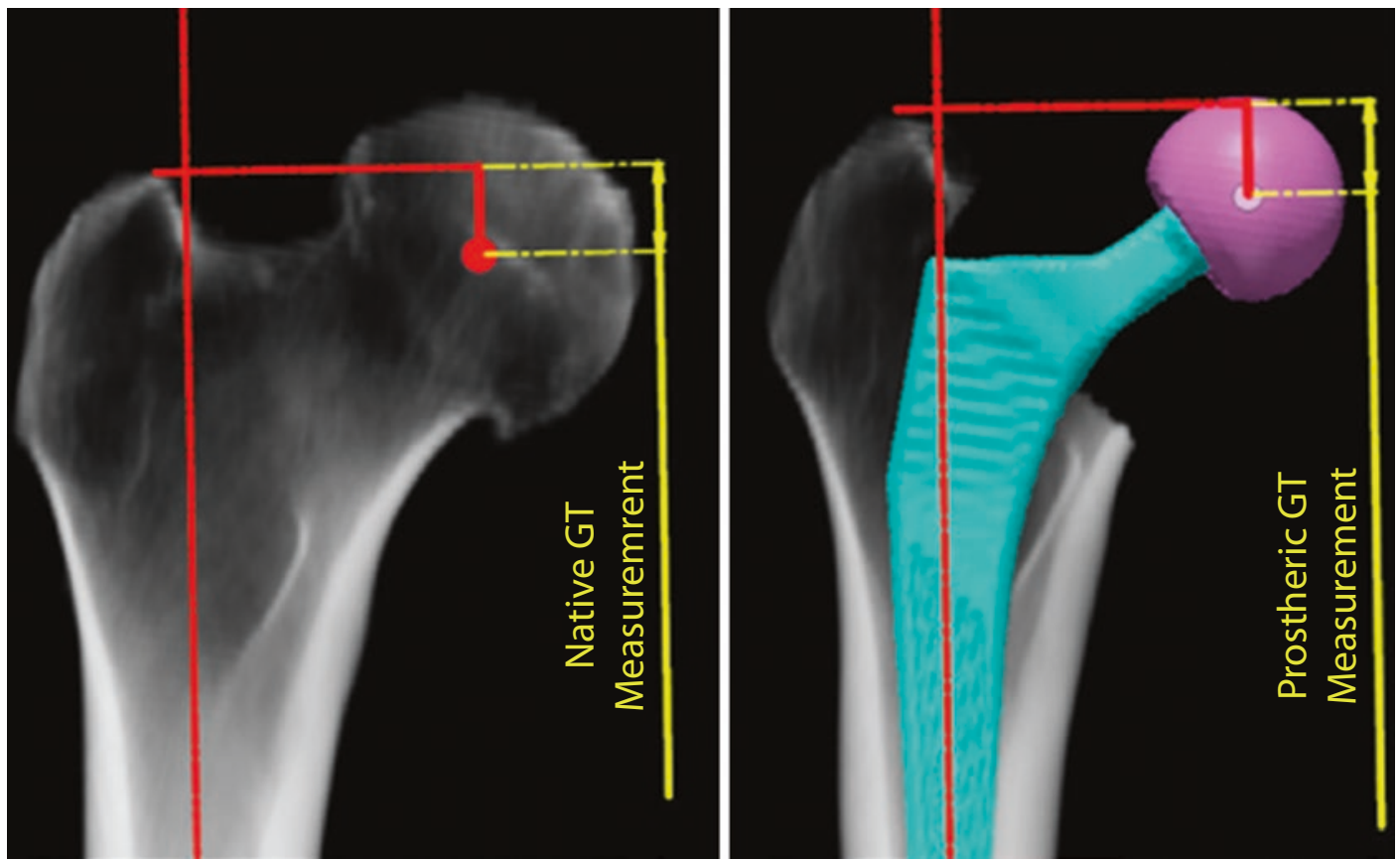

Fig. 9.6 Native and prosthetic greater trochanter measurement

\section{References}

1. Merle C, Waldstein W, Pegg E, Streit MR, Gotterbarm $\mathrm{T}$, Aldinger PR, et al. Femoral offset is underestimated on anteroposterior radiographs of the pelvis but accurately assessed on anteroposterior radiographs of the hip. J Bone Joint Surg Br. 2012;94:477-82. https://doi. org/10.1302/0301-620X.94B4.28067.

2. Khanuja HS, Vakil JJ, Goddard MS, Mont MA. Cementless femoral fixation in total hip arthroplasty. J Bone Joint Surg Am. 2011;93:500-9. https:// doi.org/10.2106/JBJS.J.00774.

3. Dorr LD, Malik A, Dastane M, Wan Z. Combined Anteversion technique for Total hip Arthroplasty. Clin Orthop Relat Res. 2009;467:119-27. https://doi. org/10.1007/s11999-008-0598-4.

4. Dimitriou D, Tsai T-Y, Kwon Y-M. The effect of femoral neck osteotomy on femoral component position of a primary cementless total hip arthroplasty. Int Orthop. 2015;39:2315-21. https://doi.org/10.1007/ s00264-015-2739-1.

5. Henckel J, Holme TJ, Skinner JA, Hart AJ. 3D-printed patient-specific guides for hip Arthroplasty. J Am Acad Orthop Surg. 2018;26:e342-8. https://doi. org/10.5435/JAAOS-D-16-00719.

6. Pierrepont J, Stambouzou C, Miles B, O'Connor P, Ellis A, Molnar R, et al. Patient-specific component alignment in total hip arthroplasty. Reconstr Rev. 2016;6:27-33. https://doi.org/10.15438/rr.6.4.148.
7. Ito H, Tanaka S, Tanaka T, Oshima H, Tanaka S. A patient-specific instrument for femoral stem placement during total hip arthroplasty. Orthopedics. 2017;40:e3747. https://doi.org/10.3928/01477447-20161108-06.

8. Schneider AK, Pierrepont JW, Hawdon G, McMahon S. Clinical accuracy of a patient-specific femoral osteotomy guide in minimally-invasive posterior hip arthroplasty. Hip Int. 2018;28:636-41. https://doi. org/10.1177/1120700018755691.

9. Huppertz A, Lembcke A, Sariali EH, Durmus T, Schwenke C, Hamm B, et al. Low dose computed tomography for 3D planning of total hip arthroplasty: evaluation of radiation exposure and image quality. $\mathrm{J}$ Comput Assist Tomogr. 2015;39:649-56. https://doi. org/10.1097/RCT.0000000000000271.

10. Schauer DA, Linton OW. Ionizing radiation exposure oh the population of the United States. Med Phys. 2009;36:5375. https://doi.org/10.1118/1.3245881.

11. Yang L, Zheng Z, Chen W, Wang J, Zhang Y. Femoral neck osteotomy guide for total hip arthroplasty. BMC Surg. 2015;15:1-6. https://doi.org/10.1186/ s12893-015-0015-3.

12. Pierrepont J, Riddell W, Miles B, Baré J, Shimmin A. Clinical accuracy of a patient-specific guide for delivering a planned femoral neck osteotomy. Orthop Proc. 2016;98-B:131.

13. Bare J, Selim J, Stambouzou C, Pierrepont J, McMahon S, Shimmin A. Clinical accuracy of a patient specific femoral neck osteotomy guide. Liverpool: Br Orthop Assoc Ann Congr; 2016. 
14. Reitman R, Pierrepont J, Shimmin A, McMahon S, Kerzhner E. Accurate reproduction of femoral Centre of rotation using $3 \mathrm{~d}$ templating and a PSI guide. Orthop Proc. 2017;99:109.

15. Pierrepont J, Miles B, Walter L, Marel E, McMahon S, Solomon M, et al. Sizing accuracy of the trinity 3D planning software for total hip replacement conclusions. In: Paris: Int Congr Jt Reconstr; 2015.

16. Flecher X, Ollivier M, Argenson JN. Lower limb length and offset in total hip arthroplasty. Orthop Traumatol Surg Res. 2016;102:S9-20. https://doi. org/10.1016/j.otsr.2015.11.001.

17. Hofmann AA, Skrzynski MC. Leg-length inequality and nerve palsy in total hip arthroplasty: a lawyer awaits. Orthopedics. 2000;23:943-4.

18. Konyves A, Bannister GC. The importance of leg length discrepancy after total hip arthroplasty. J Bone Joint Surg Br. 2005;87:155-7. https://doi. org/10.1302/0301-620X.87B2.14878.

19. Woolson ST, Hartford JM, Sawyer A. Results of a method of leg-length equalization for patients undergoing primary total hip replacement. J Arthroplast. 1999;14:159-64.

20. Renkawitz T, Weber T, Dullien S, Woerner M, Dendorfer S, Grifka J, et al. Gait \& posture leg length and offset differences above $5 \mathrm{~mm}$ after total hip arthroplasty are associated with altered gait kinematics. Gait Posture. 2016;49:196-201. https://doi. org/10.1016/j.gaitpost.2016.07.011.

21. Sykes A, Hill J, Orr J, Humphreys P, Rooney A, Morrow E, et al. Patients' perception of leg length discrepancy post total hip arthroplasty. Hip Int. 2015;25:452-6. https://doi.org/10.5301/hipint.5000276.
22. Mahmood SS, Mukka SS, Crnalic S, Sayed-Noor AS. The influence of leg length discrepancy after total hip arthroplasty on function and quality of life: a prospective cohort study. J Arthroplast. 2015;30:163842. https://doi.org/10.1016/j.arth.2015.04.012.

23. Li J, McWilliams AB, Jin Z, Fisher J, Stone MH, Redmond AC, et al. Unilateral total hip replacement patients with symptomatic leg length inequality have abnormal hip biomechanics during walking. Clin Biomech (Bristol, Avon). 2015;30:513-9. https://doi. org/10.1016/j.clinbiomech.2015.02.014.

24. Sariali E, Klouche S, Mouttet A, Pascal-Moussellard $H$. The effect of femoral offset modification on gait after total hip arthroplasty. Acta Orthop. 2014;85:1237. https://doi.org/10.3109/17453674.2014.889980.

25. Clement ND, S Patrick-Patel R, MacDonald D, Breusch SJ. Total hip replacement: increasing femoral offset improves functional outcome. Arch Orthop Trauma Surg. 2016;136:1317-23. https://doi. org/10.1007/s00402-016-2527-4.

26. Al-amiry B, Mahmood S, Krupic F. Leg lengthening and femoral-offset reduction after total hip arthroplasty: where is the problem - stem or cup positioning? Acta Radiol. 2017;58:1125-31. https:// doi.org/10.1177/0284185116684676.

27. Mahmood SS, Mukka SS, Crnalic S, Sayed-Noor AS. Association between changes in global femoral offset after total hip arthroplasty and function, quality of life, and abductor muscle strength a prospective cohort study of 222 patients. Acta Orthop. 2016;87:36-41. https://doi.org/10.3109/17453674.20 15.1091955 .

Open Access This chapter is licensed under the terms of the Creative Commons Attribution 4.0 International License (http://creativecommons.org/licenses/by/4.0/), which permits use, sharing, adaptation, distribution and reproduction in any medium or format, as long as you give appropriate credit to the original author(s) and the source, provide a link to the Creative Commons license and indicate if changes were made.

The images or other third party material in this chapter are included in the chapter's Creative Commons license, unless indicated otherwise in a credit line to the material. If material is not included in the chapter's Creative Commons license and your intended use is not permitted by statutory regulation or exceeds the permitted use, you will need to obtain permission directly from the copyright holder.

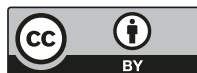

